\title{
Una Alternativa para la Simulación Numérica del Comportamiento Térmico en Régimen Transitorio de Flujo de Gas en Redes de Ductos
}

\author{
Arturo J. Ortega, Luis F.G. Pires y Angela O. Nieckele \\ Pontificia Universidad Católica de Río de Janeiro, Departamento de Ingeniería Mecánica, Núcleo de \\ Simulación Termo-hidráulica de Ductos-SIMDUT, Rua Marques de Sao Vicente 225, Gávea, \\ 22453-900 Rio de Janeiro, RJ-Brasil \\ (e-mail: arturo@simdut.com.br; Ipires@simdut.com.br; nieckele@puc-rio.br)
}

\begin{abstract}
Resumen
En este trabajo se desarrolló un nuevo y práctico código computacional, para simular el comportamiento térmico en régimen transitorio de flujo de gas en redes de ductos, en presencia de varios tipos de componentes como válvulas, compresores, intercambiadores de calor, y bifurcaciones. El modelo matemático está basado en los principios de conservación de masa, cantidad de movimiento lineal y energía, además de ecuaciones constitutivas para los diversos componentes. La solución de las ecuaciones de conservación fue realizada usando el método de los volúmenes finitos. Diversos casos de estudio fueron analizados utilizando circuitos con diferentes tipos de bifurcación y varias combinaciones de puntos de admisión y descarga, localizados a lo largo de las líneas de los gasoductos. Los resultados de las pruebas fueron comparados con datos obtenidos usando un software comercial, presentando excelente concordancia.
\end{abstract}

Palabras clave: redes de ductos, flujo de gas térmico, régimen transiente, simulación numérica

\section{An Alternative to Numerical Simulation of Thermal Transient Behavior Gas Flow in Pipeline Networks}

\begin{abstract}
In this work a new and practical computational code to simulate thermal transient gas flow in pipeline networks with presence of several types of components as valves, compressors, heat exchangers, and pipe bifurcations, was developed The mathematical model is based on the conservation principles of mass, linear momentum and energy, plus the constitutive equations for each component. The conservation equations solution was done using the finite volume method. Several study cases were analyzed using pipe networks with different types of bifurcation and different combination of supply and delivery points located along the network. The tests results were compared with data obtained using commercial software showing excellent agreement.
\end{abstract}

Keywords: pipeline network, thermal gas flow, transient regime, numerical simulation 


\section{INTRODUCCIÓN}

Cada vez se están diseñando, construyendo y operando sistemas de gasoductos más complejos. Debido a la importancia de esos sistemas, se están realizando esfuerzos para desarrollar modelos matemáticos y métodos numéricos, cada vez más generales y eficientes, dirigidos a simular computacionalmente esos gasoductos con la finalidad de maximizar sus operaciones, entre otros aspectos.

Diversos son los trabajos relacionados con la simulación de gasoductos, entre los cuales se puede mencionar el texto de Wylie y Streeter (1993), donde se describen las metodologías de implementación de componentes y acoplamientos. Nieckele et al. (2001), presentaron un modelo para la simulación del movimiento de pigs. Las ecuaciones de conservación fueron combinadas con la ecuación del pig y estas, a su vez, resueltas utilizando el método de diferencias finitas. Barrera et al. (2006), presentaron un análisis de la capacidad térmica de las paredes y de las capas de revestimiento durante un régimen transitorio y térmico. Mahgerefteh et al (2006), presentaron un modelo que simula el comportamiento del fluido durante la ruptura de una línea de ducto usando el método de las características, demostrando la importancia de considerar la complejidad de la red cuando se simula este tipo de falla. Abhulimen y Susu (2007), desarrollaron un modelo para la detección de fugas en líneas de ductos. Aquí la red es dividida en pequeñas subredes las cuales son resueltas usando el método de las características. Herrán-González et al. (2008), presentaron un modelo para la distribución de gas en redes de ductos usando el método de las características, demostrando que la caída de presión tiene una fuerte dependencia con el factor de inclinación.

El objetivo del presente trabajo consiste en presentar una metodología para simular el comportamiento térmico en régimen transitorio de flujo gas en redes de ductos. La metodología es validada a través de comparaciones con datos obtenidos de software comerciales.

\section{MODELADO MATEMÁTICO}

En general, flujos de gases a través de ductos son considerados como unidimensionales, además el gas es considerado casi ideal, i.e. la masa específica $\rho=P W /(z \Re T)$, donde $P$ y $T$ son la presión y la temperatura respectivamente, $\Re$ es la constante universal, $W$ es el peso molecular y $z$ es el factor de compresibilidad. Para el cálculo del factor de compresibilidad se utilizó la ecuación de estado CNGA (California Natural Gas Association), Pipeline Studio (2004). Las otras propiedades termodinámicas como la viscosidad $\mu$, la conductividad térmica $k$ y el calor específico a presión constante $c_{p}$ son considerados constantes, así como las propiedades relacionadas al ducto, el módulo de elasticidad $E$ y el coeficiente de Poisson $v$.

Tomando en consideración la compresibilidad del fluido y la variación del área $A$ del ducto a lo largo del mismo, debido a los diferentes diámetros $D$ y espesores $\delta$ así como también por su dilatación debido a la presión, el principio de conservación de masa aplicada a un volumen de control infinitesimal el cual puede presentar una inclinación $\theta$ con relación a la horizontal es:

$$
\frac{\partial P}{\partial t}+V \frac{\partial P}{\partial x}+\frac{\rho a^{2}}{\xi} \frac{\partial V}{\partial x}+\frac{\rho a^{2}}{\xi} \frac{V}{A} \frac{\partial A}{\partial x}-\frac{\rho a^{2} \beta}{\xi}\left(\frac{\partial T}{\partial t}-V \frac{\partial T}{\partial x}\right)=0
$$

donde $a=\partial P /\left.\partial \rho\right|_{T}$ es la velocidad del sonido isotérmica, $\beta=(-1 / \rho) \partial \rho /\left.\partial T\right|_{\mathrm{P}}$ es el coeficiente de expansión térmica y el parámetro $\xi$ es $\xi=1+\rho a^{2} 2\left(1-v^{2}\right) D /(2 \delta E)$, Nieckele et al. (2001). El principio de conservación de cantidad de movimiento lineal es expresado como:

$$
\frac{\partial V}{\partial t}+V \frac{\partial V}{\partial x}=-\frac{1}{\rho} \frac{\partial P}{\partial x}-\frac{f}{2} \frac{V|V|}{D}-g \operatorname{sen} \theta
$$

donde $V$ es la velocidad del fluido, $g$ es la aceleración de la gravedad y $f$ es el factor de fricción, el 
cual depende del número de Reynolds, $\operatorname{Re}=\rho \vee D / \mu$. Para el cálculo del factor de fricción la expresión analítica de Hagen Pousseiulle $f=64 /$ Re es usada en el caso que el régimen sea laminar y en el caso que el régimen sea turbulento, la correlación de Millar $f=0.25\left\{\log \left[(d / D) / 3.7+5.75 / \operatorname{Re}^{0.9}\right]\right\}^{-2}$, basada en la rugosidad $\varepsilon$ y numero de Reynolds, es usada, Fox et al. (2006). La distribución de la temperatura es obtenida a partir de la ecuación de conservación de energía, dependiendo del coeficiente global de transferencia de calor $U_{e}$ y de la temperatura ambiente $T_{0}$ :

$$
\frac{\partial T}{\partial t}+V \frac{\partial T}{\partial x}=\frac{\beta T}{\rho c_{p}}\left(\frac{\partial P}{\partial t}+V \frac{\partial P}{\partial x}\right)+\frac{f}{2 c_{p}} \frac{V^{2}|V|}{D}-\frac{4 U_{e}}{\rho c_{p} D}\left(T-T_{O}\right)
$$

Una relación de los modelos matemáticos de los componentes pueden ser encontrados en Nieckele et al. (2007). Por ejemplo, las curvas características del compresor representan el comportamiento de la carga isentrópica $H$ y de la eficiencia isentrópica $\eta$ como una función del caudal del gas $Q$. Adicionalmente, la cantidad de trabajo por unidad de masa $H$ es definido como $H=\left(T_{d}-T_{s}\right) \quad \eta z_{m} C_{p}$, donde los subíndices $s, d$ y $m$ están relacionados con los valores en la succión, descarga y valor medio del componente. La temperatura en la descarga del compresor $T_{d}=T_{s}+T_{s} / \eta\left[\left(P_{s} / P_{d}\right)^{(R /(W c p)}-1\right]$ representa el natural incremento de temperatura como consecuencia del proceso de compresión. Para el modelado matemático de las válvulas intermediarias y de alivio fueron usadas las normas ISA S75.01-S75.02 (Hutchison, 1976). Los intercambiadores de calor son dispositivos que sirven para aumentar o disminuir la temperatura del flujo de gas. El calor intercambiado es igual a la diferencia de entalpías entre la entrada y la salida del intercambiador. La caída de presión en este componente es definido como $K=\left(P_{s}^{2}-P_{d}^{2}\right) / Q_{\text {std }}^{2}$, Pipeline Studio (2004), donde $Q_{\text {std }}$ es el caudal volumétrico en condiciones estándar.

\section{MÉTODO NUMÉRICO}

La solución de las ecuaciones gobernantes del fluido es realizada mediante el método de los volúmenes finitos (Patankar, 1980). La derivada espacial es aproximada usando el método de las diferencias centrales y una aproximación completamente implícita es adoptada para la integración en el tiempo. Se considera el acoplamiento de las ecuaciones gobernantes, las cuales son solucionadas simultáneamente utilizando un algoritmo hepta-diagonal directo.

Para la solución numérica de las conexiones de ductos se considera una presión y temperatura común en el nodo que representa el empalme. La presión es determinada mediante un balance de masa acoplando la ecuación de cantidad de movimiento. Similarmente la temperatura es calculada mediante un balance de energía. En el caso de los componentes, los principios de las ecuaciones de conservación también son respetados. Estas ecuaciones de conservación combinadas con el modelo matemático del componente definen las condiciones de contorno de los ductos conectados a este, Wylie y Streeter (1993).

Una vez obtenida el conjunto de ecuaciones algebraicas para cada volumen de control y definidas las condiciones de contorno para cada ducto, estas son resueltas en forma secuencial por cada ducto a lo largo del tiempo.

\section{RESULTADOS Y DISCUSIÓN}

Con la intención de validar el programa desarrollado, NetGasSim, fueron ejecutados diferentes ensayos, algunos de las cuales son mostradas en este artículo. Los resultados obtenidos fueron corroborados con resultados del software TGNET de Pipeline Studio.

En la figura 1 se observa una serie de ductos de 16 in con extensión de 40 y $30 \mathrm{~km}$, dos de los cuales están en paralelo. En la entrada de la red es impuesto un caudal másico de $0.7 \mathrm{~kg} / \mathrm{s}$ a $50{ }^{\circ} \mathrm{C}$ y en la salida es considerada la presión atmosférica. Los resultados obtenidos para la distribución de presión, temperatura y caudal másico a lo largo de la red son mostrados en las figuras $2 a, 2 b$ y $2 c$ 
respectivamente. Se puede observar la excelente concordancia con la previsión del software TGNET, con errores inferiores a $0.1 \%$.

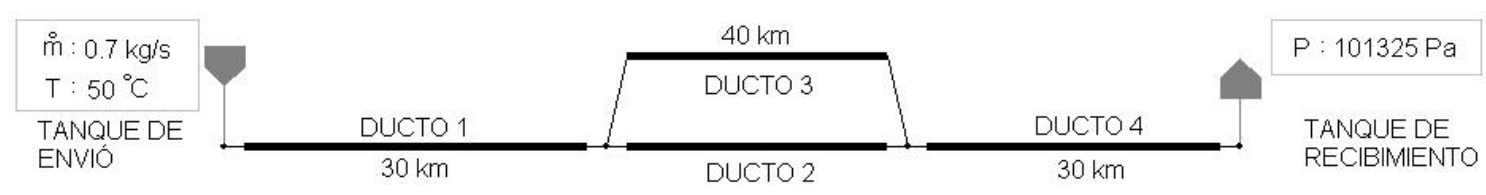

Fig. 1: Configuración de ductos en paralelo

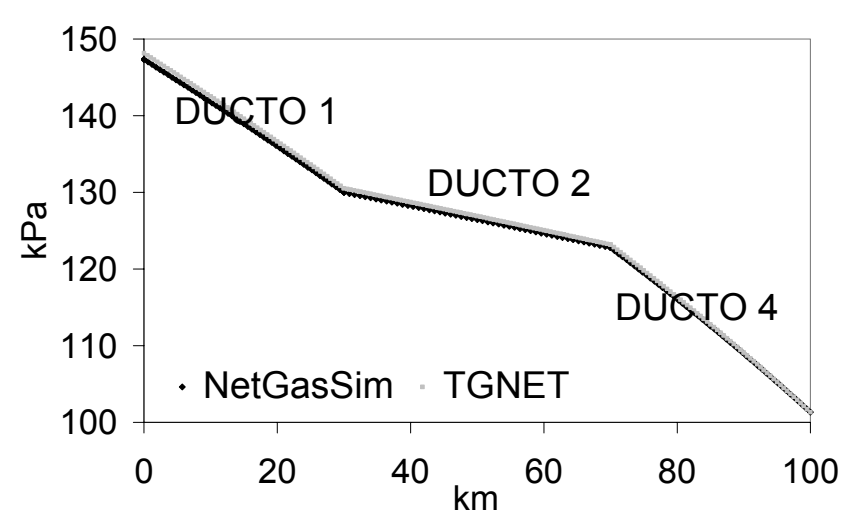

(a) Distribución de presión

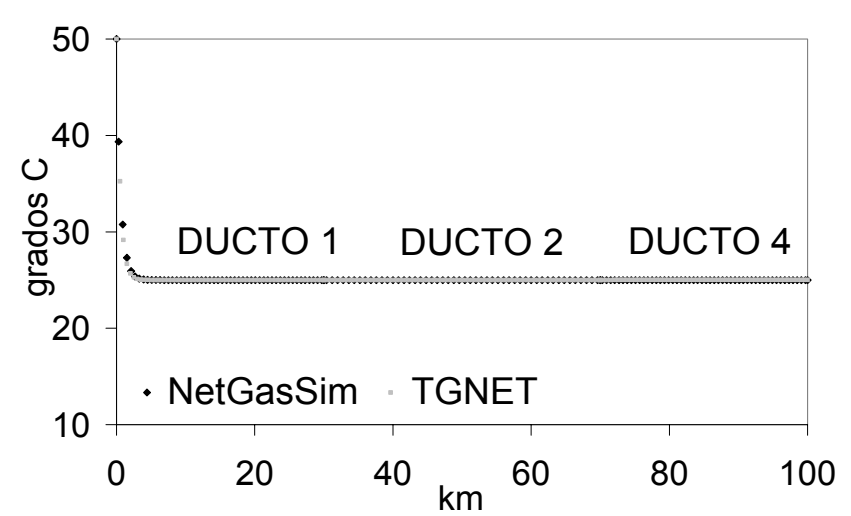

(b) Distribución de temperatura

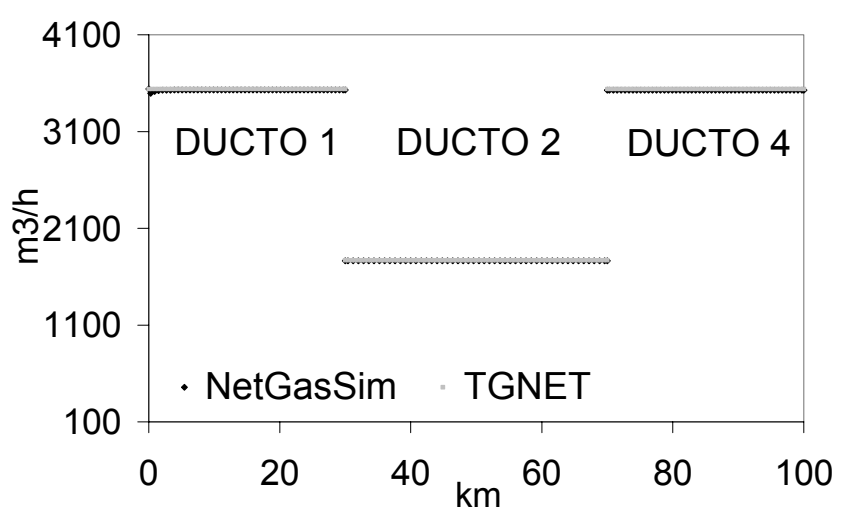

(c) Distribución del caudal másico

Fig. 2: Ductos en paralelo

Un segundo ensayo fue realizado en el gasoducto llamado GASFOR, perteneciente al sistema de distribución de gas brasileño (figura 3), el cual posee un diámetro de 12 in en los primeros $213 \mathrm{~km}$ y un diámetro de 10 in en los restantes $170 \mathrm{~km}$. Las condiciones de contorno son: en la ciudad de Guamare es establecida una presión de $8927.31 \mathrm{kPa}$ y una temperatura de $35^{\circ} \mathrm{C}$. En el $\mathrm{km} 213$ de la línea se encuentra la estación de compresión de Aracati donde es establecida una presión de descarga de $9417.64 \mathrm{kPa}$. Este gasoducto posee una serie de puntos de retirada a lo largo de la línea, los cuales originan que el flujo de masa varíe de $16.98 \mathrm{~kg} / \mathrm{s}$ en la entrada para aproximadamente $0.01 \mathrm{~kg} / \mathrm{s}$ en la ciudad de Pecem.

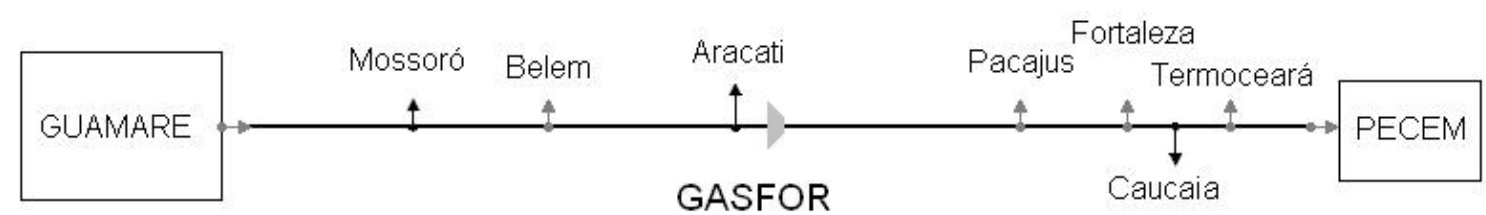

Fig.3: Configuración GASFOR

Los resultados de las simulaciones, usando los dos software, para la distribución de presión, temperatura y caudal másico son mostrados en las figuras $4 a, 4 b$ y $4 c$ respectivamente. La excelente 
concordancia entre los dos simuladores confirma la validez de la presente metodología. Nuevamente errores inferiores a $0.1 \%$ fueran encontrados para a presión e inferiores a $5 \%$ para a temperatura.

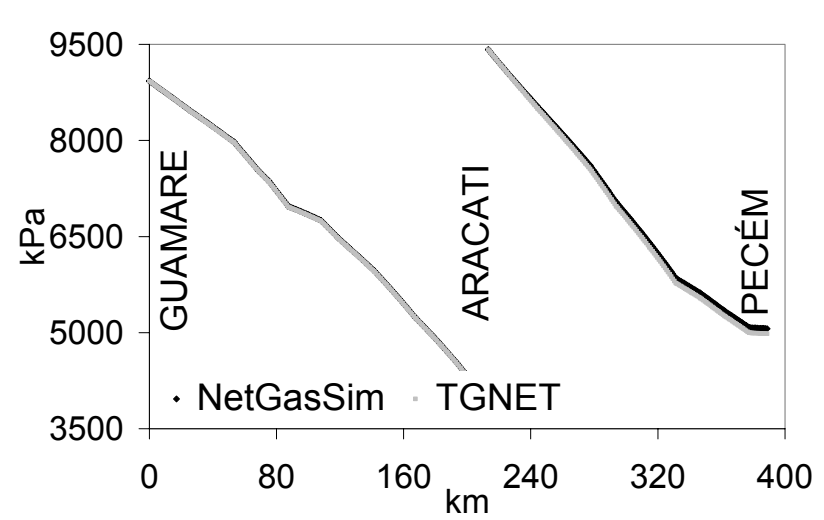

(a) Distribución de presión

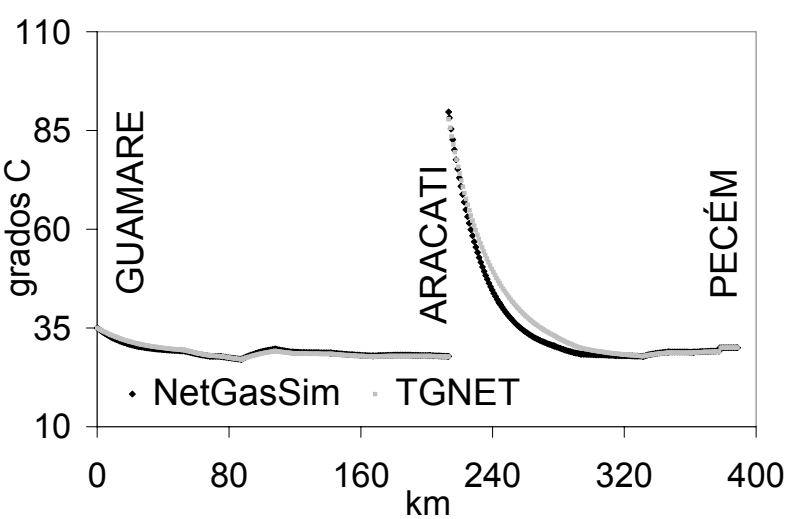

(b) Distribución de temperatura

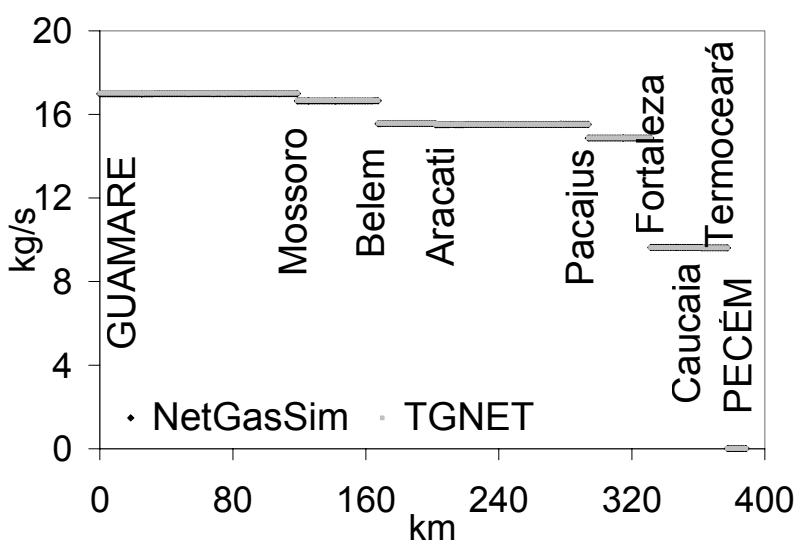

(c) Distribución del caudal másico

Fig.4: GASFOR

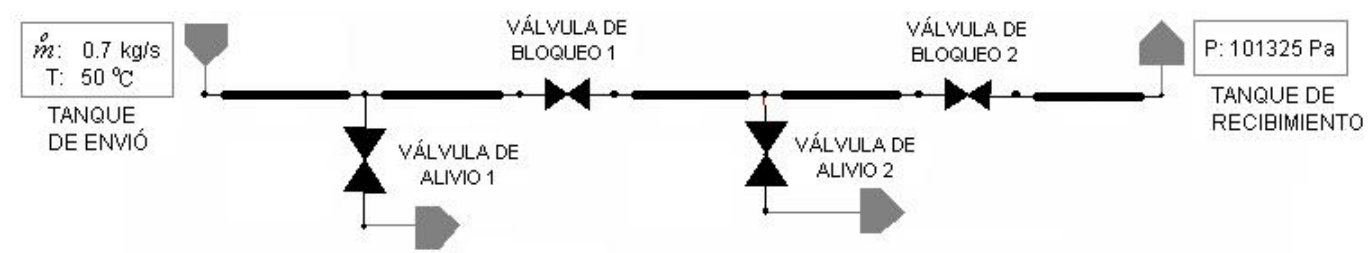

Fig.5: Configuración del análisis del régimen transitorio
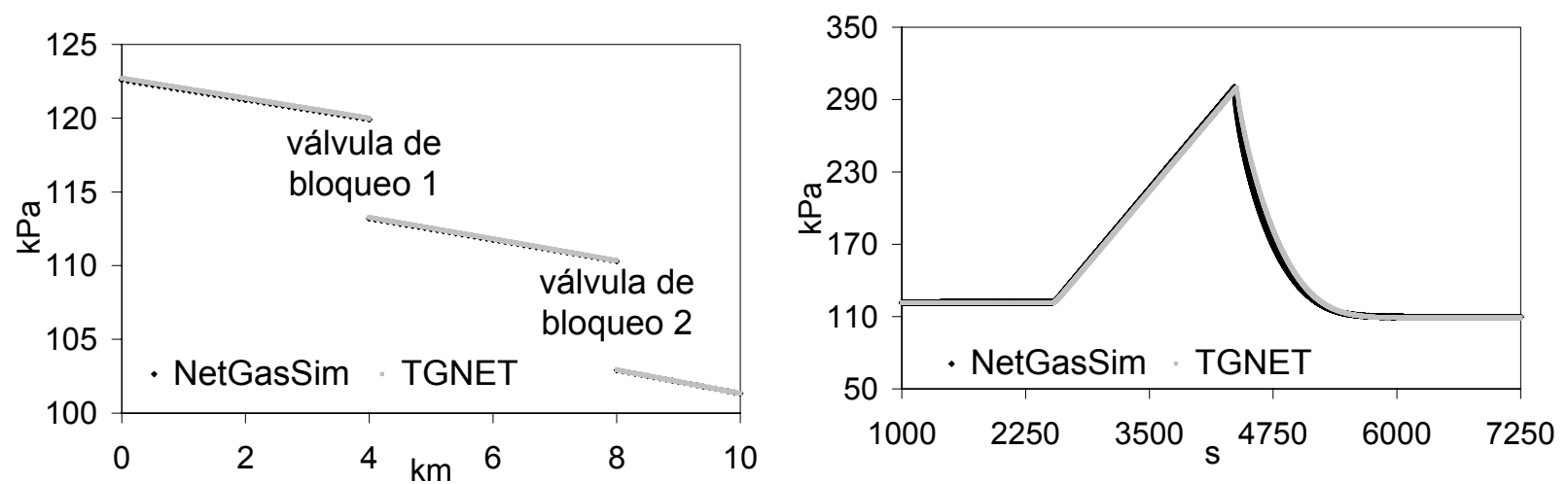

(a) Distribución de presión -régimen permanente (b) Válvula de alivio durante el régimen transitorio

Fig.6: Análisis del régimen transitorio 
La figura 5 ilustra la configuración utilizada para analizar el régimen transitorio durante la actuación de una válvula de alivio, en una línea de $10 \mathrm{~km}$ y 16 in de diámetro, en la cual están distribuidas uniformemente dos válvula de bloqueo y dos de alivio. En la entrada de la línea principal es impuesto un caudal de masa de $0.7 \mathrm{~kg} / \mathrm{s}$ a $50^{\circ} \mathrm{C}$ y en la salida es considerada la presión atmosférica. En las válvulas de alivio san establecidas las presiones de ajuste de $300 \mathrm{kPa}$ con un tiempo de abertura total de $3 \mathrm{~s}$.

Después de alcanzar el régimen permanente (figura 6a), la válvula de bloqueo 2 comienza a cerrar en el instante de 2500s, cerrando totalmente después de 20s. La figura $6 \mathrm{~b}$ muestra la variación de la presión con el tiempo en la válvula de alivio 1 (la válvula de bloqueo 2 no abrió). Nuevamente, se observa la excelente concordancia entre los dos software (errores inferiores a $0.5 \%$ ).

\section{CONCLUSIONES}

De acuerdo a los resultados obtenidos en las simulaciones presentadas, se puede afirmar que el modelado, la técnica y el código computacional desarrollados representan una buena herramienta para la simulación de flujo de gas en redes de ductos, el cual muestra funcionalidades y resultados semejantes a los de conocidos software presentes en el mercado, los errores obtenidos fueron de prácticamente cero por ciento en comparación al software comercial. En futuras ampliaciones del trabajo se considerara la implementación de otros componentes, el análisis del régimen transitorio originado por otros componentes y la minimización del tiempo computacional.

\section{AGRADECIMIENTOS}

Al apoyo financiero proporcionado por ANEEL (Agencia Nacional de Energía Eléctrica - Brasil) y CNPq (Consejo Nacional de Desarrollo Científico y Tecnológico - Brasil).

\section{REFERENCIAS}

Abhulimen, K.E. y A.A. Susu; Modeling Complex Pipeline Network Leak Detection Systems, Trans IChem E - Part B - Process Safety and Environmental Protection: 85(B6), 579-598 (2007).

Barrera, J.J., A.O. Nieckele y L.F.A. Azevedo; Analysis of the Transient Cooldown of Sub-Sea Pipelines, 6th International Pipeline Conference, Calgary, Canada, 25 al 29 de septiembre (2006).

Fox, R.W., A.T. McDonald y P.J. Pritchard; Introdução a Mecânica dos Fluidos, Ed LTD, 6ª Ed. (2006)

Herrán-Gonzáles, A., J.M. De La Cruz, B. De Andrés-Toro y J.L. Risco-Martín; Modeling and Simulation of a Gas Distribution Pipeline Network, Applied Mathematical Modeling (2008), www.sciencedirect.com. Acceso: 18 de Septiembre (2008).

Hutchison, J.W.; ISA Handbook of Control Valves, $2^{a}$ ed., Instrument Society of America, USA (1976).

Mahgerefteh, H., A. Oke y O. Atti; Modeling Outflow Following Rupture in Pipelines Networks, Journal of Chemical Engineering Science: 61, 1811-1818 (2006).

Nieckele, A.O., A.M.B. Braga y L.F.A Azevedo; Transient Pig Motion through Gas and Liquid Pipelines, Journal of Energy Resources Technology: 123, 260-269 (2001).

Nieckele, A.O., L.F.G. Pires y A.J. Ortega; Um Modelo para Análise de Escoamento Transiente Térmico em Gasodutos e seus Componente, Rio Pipeline Conference \& Exposition 2007, Rio de Janeiro, Brasil, 2 al 4 de Octubre (2007).

Patankar, S.V.; Numerical Heat Transfer and Fluid Flow, Hemisphere Publ. Corp., NY, USA (1980).

Pipeline Studio, User Guide, versión 2.5 (2004).

Wylie, E.B. y V.L. Streeter; Fluid Transients in Systems, Prentice Hall, New Jersey, USA (1993). 